\title{
Dynamical Strategy to Control the Accuracy of the Nonlinear Bio-Mathematical Model of Malaria Infection
}

\author{
Samad Noeiaghdam ${ }^{1,2 *(D)}$ and Sanda Micula ${ }^{3}$ (D) \\ 1 Department of Applied Mathematics and Programming, South Ural State University, Lenin Prospect 76, \\ 454080 Chelyabinsk, Russia \\ 2 Industrial Mathematics Laboratory, Baikal School of BRICS, Irkutsk National Research Technical University, \\ 664074 Irkutsk, Russia \\ 3 Department of Mathematics, Babeş-Bolyai University, 400084 Cluj-Napoca, Romania; \\ smicula@math.ubbcluj.ro \\ * Correspondence: snoei@istu.edu or noiagdams@susu.ru
}

Citation: Noeiaghdam, S.; Micula, S. Dynamical Strategy to Control the Accuracy of the Nonlinear Bio-Mathematical Model of Malaria Infection. Mathematics 2021, 9, 1031. https://doi.org/10.3390/ math 9091031

Academic Editors: M. Victoria Otero-Espinar

Received: 18 March 2021

Accepted: 28 April 2021

Published: 2 May 2021

Publisher's Note: MDPI stays neutral with regard to jurisdictional claims in published maps and institutional affiliations.

Copyright: (C) 2021 by the authors. Licensee MDPI, Basel, Switzerland. This article is an open access article distributed under the terms and conditions of the Creative Commons Attribution (CC BY) license (https:/ / creativecommons.org/licenses/by/ $4.0 /)$.

\begin{abstract}
This study focuses on solving the nonlinear bio-mathematical model of malaria infection. For this aim, the HATM is applied since it performs better than other methods. The convergence theorem is proven to show the capabilities of this method. Instead of applying the FPA, the CESTAC method and the CADNA library are used, which are based on the DSA. Applying this method, we will be able to control the accuracy of the results obtained from the HATM. Also the optimal results and the numerical instabilities of the HATM can be obtained. In the CESTAC method, instead of applying the traditional absolute error to show the accuracy, we use a novel condition and the CESTAC main theorem allows us to do that. Plotting several $\hbar$-curves the regions of convergence are demonstrated. The numerical approximations are obtained based on both arithmetics.
\end{abstract}

Keywords: malaria infection; discrete stochastic arithmetic; CESTAC method; CADNA library; homotopy analysis transform method

\section{Introduction}

Modeling different phenomena, solving the model by efficient methods and also control and adjust their parameters are one of the important topics of bio-mathematics which have direct relation with human life. Thus, many mathematicians have been focused on various mathematical models such as energy supply-demand model [1], SIR epidemic model [2], HIV infection [3-5], model of smoking habit [6], bovine babesiosis disease [7], model of computer viruses [8-10] and many other models. Malaria is one of the important infections for which various models have been proposed to control, prevent and transmit. Due to the high prevalence of this infection in Africa, most studies have been performed on data obtained from Africa. According to the World Health Organization, in 2019, approximately 409,000 people died of malaria [11].

HATM is one the semi analytical methods for solving different linear and nonlinear problems. This method was presented by Liao [12-14] for the first time and because of some of flexibilities, this method has been applied by many researchers. Solving integral equations [15-18], system of equations $[6,10]$ and engineering problems $[19,20]$ are only some of the applications of this method. In this method, we have some freedom to choose the initial values, the auxiliary functions and the auxiliary parameters. One of these parameters is $\hbar$, which is used to adjust and control the convergence region of the HATM. Using this parameter we can plot some $\hbar$-curves and after that we will be able to find the convergence region. 
We should know that in the mentioned researches and many other papers, the computations are based on the FPA and in order to show the efficiency of the method, we apply the traditional absolute error as follows

$$
\left|f(x)-f_{n}(x)\right|<\varepsilon, \text { or }\left|f_{n}(x)-f_{n-1}(x)\right|<\varepsilon,
$$

where $f(x)$ and $f_{n}(x)$ are exact and approximate solutions of the problem and $\varepsilon$ is a small positive value. However, in this case, we should know the exact solution and the optimal value of $\varepsilon$, and this is one of the big bugs of this relation. If we choose value $\varepsilon$ by chance, for large values we will have only one or two iterations without providing accurate results and if we have small values of $\varepsilon$, we will have extra iterations without improving the accuracy [21]. Thus, we introduce a new arithmetic, which is the DSA [22]. For this aim, we use the CESTAC method and instead of the usual softwares we apply the CADNA library [23]. Moreover, instead of applying condition (1), we use a novel condition as follows

$$
\left|f_{n}(x)-f_{n-1}(x)\right|=@ .0,
$$

where $f_{n}(x)$ and $f_{n-1}(x)$ are two successive approximations and @.0 denotes the informatical zero $[24,25]$. This sign can be produced only in the CESTAC method and by the CADNA library. This library should be done on the Linux operating system and all CADNA codes should be written by $\mathrm{C}, \mathrm{C}++$, FORTRAN or ADA codes [26,27]. Applying the CESTAC method, we can find the optimal iteration of the method, the optimal approximation, the optimal error and some numerical instabilities [27-29]. We will also be able to cancel the extra iterations. The main theorem of the CESTAC method will aid us in applying the new condition instead of the previous one. In this theorem, we will show that the number of common significant digits for two successive approximations is almost equal to the number of common significant digits for exact and approximate solutions [21]. Recently, this method has been applied to validate the results of some numerical methods to solve the mathematical and engineering problems such as numerical integration rules [30,31], homotopy analysis method [32-34], homotopy perturbation method [35], Adomian decomposition method [36], collocation method [37-39], solving fuzzy problems [37,40,41], load leveling problem [42], reverse osmosis model [43] and many others [44]. For more information about the DSA, the CESTAC method and the CADNA library please see [21].

In this paper, at first we will focus on solving the nonlinear model of malaria infection (3) by using the HATM. The convergence theorem is also proved to show the abilities of this method. The CESTAC method and the CADNA library are applied to validate the numerical results. The main theorem of the CESTAC method is proved. This theorem will help us to apply the termination criterion (2) instead of (1). The numerical results are obtained for both the FPA and the DSA. Some $\hbar$-curves are also plotted to show the convergence regions. Based on the CESTAC method, the optimal iteration, the optimal approximation, the optimal error and the numerical instabilities are found. 


\section{Model Description}

In this paper, we will focus on the following nonlinear model of malaria infection [45]:

$$
\begin{aligned}
& \frac{d I}{d t}=m P_{H} \beta_{H} S Z-\left(\varepsilon+\mu_{H}\right) I, \\
& \frac{d R}{d t}=\varepsilon I+k A-\left(\theta m P_{H} \beta_{H} Z+\tau+\mu_{H}\right) R, \\
& \frac{d A}{d t}=\theta m P_{H} \beta_{H} R Z-\left(k+\mu_{H}\right) A, \\
& \frac{d Y}{d t}=P_{H} \beta_{Z}(I+\sigma A) X-\left(\xi+\mu_{v}\right) Y, \\
& \frac{d Z}{d t}=\xi Y-\mu_{v} Z,
\end{aligned}
$$

where an infectious mosquito ' $Z$ ', will bite ' $S$ ' usceptible hosts and make them ' $I$ 'nfected. We know that infected hosts before reversion to fully susceptible at rate ${ }^{\prime} \tau^{\prime}$ can have level of temporary immunity $(1-\theta$, where $0 \leq \theta \leq 1)$. However, we should note that hosts can become ' $A$ 'symptomatically infected during this ' $R$ ' ecovered state. Symptomatic infection is a temporary condition that may have different transmission potentials to $(\sigma)$ carriers, after which people return to the improved group at a rate of $\kappa$. In this model, considering that a fixed human population is assumed, human mortality is compensated by births and we get $S=1-(I+R+A)$. Sensitive $(X)$ vectors begin to become infected after being bitten by an infected infectious host, but they are not yet infected $(Y)$, but after the extrinsic incubation period $\left(\frac{1}{\xi}\right)$, become infectious $(Z)$. We apply $m$ to show the ratio of vector-to-hosts and vectors typically outnumber hosts. In order to balance the births and deaths rate we define the stable vector $\left(\mu_{v}\right)$ and we get $X=1-(Y+Z)$. The parameters and values are listed in Table 1.

Table 1. List of parameters and functions [45].

\begin{tabular}{clc}
\hline Parameters & Meaning & Values \\
\hline$\beta_{z}$ & Transmission coefficient (vectors $\rightarrow$ hosts) $=$ bite rate $\times$ transmission probability & $0.1=\frac{1}{3} \times 0.3$ \\
$\beta_{H}$ & Transmission coefficient (hosts $\rightarrow$ vectors) $=$ bite rate $\times$ transmission probability & $0.007=\frac{1}{3} \times 0.02$ \\
$\varepsilon$ & Clearance rate of symptomatic infection & $\frac{1}{200}$ \\
$\mu_{H}$ & Birth and death rate of humans (i.e., stable population) & $\frac{1}{21900}$ \\
$k$ & Clearance rate of asymptomatic infection & $\frac{1}{200}$ \\
$\theta$ & Asymptomatic secondary infection rate & 0.5 \\
$\tau$ & Full susceptibility reversion rate & 1 \\
$\sigma$ & Adjustment factor for asymptomatic transmissibility to vector & 0.25 \\
$\mu_{v}$ & Birth (or maturation) and death rate of vectors (i.e., stable population) & 0.1 \\
$\xi$ & Rate of parasite development within vector & 0.1 \\
\hline
\end{tabular}

\section{Homotopy Analysis Transform method}

In order to apply the HATM, we combine the HAM with Laplace transformations. For this reason by using the Laplace transformation $\mathcal{L}$ for both sides of non-linear system (3) we get 


$$
\begin{aligned}
& \mathcal{L}[I(t)]=\frac{I(0)}{s}+\frac{m P_{H} \beta_{H}}{s} \mathcal{L}[Z-I Z-R Z-A Z]-\frac{\left(\varepsilon+\mu_{H}\right)}{s} \mathcal{L}[I], \\
& \mathcal{L}[R(t)]=\frac{R(0)}{s}+\frac{\varepsilon}{s} \mathcal{L}[I]+\frac{k}{s} \mathcal{L}[A]-\frac{\theta m P_{H} \beta_{H}}{s} \mathcal{L}[Z R]-\frac{\tau}{s} \mathcal{L}[R]+\frac{\mu_{H}}{s} \mathcal{L}[R], \\
& \mathcal{L}[A(t)]=\frac{A(0)}{s}+\frac{\theta m P_{H} \beta_{H}}{s} \mathcal{L}[R Z]-\frac{\left(k+\mu_{H}\right)}{s} \mathcal{L}[A], \\
& \mathcal{L}[Y(t)]=\frac{Y(0)}{s}+\frac{P_{H} \beta_{Z}}{s} \mathcal{L}[I-I Y-I Z]+\frac{P_{H} \beta_{Z} \sigma}{s} \mathcal{L}[A-A Y-A Z]-\frac{\left(\xi+\mu_{v}\right)}{s} \mathcal{L}[Y], \\
& \mathcal{L}[Z(t)]=\frac{Z(0)}{s}+\frac{\xi}{s} \mathcal{L}[Y]-\frac{\mu_{v}}{s} \mathcal{L}[Z] .
\end{aligned}
$$

In order to apply the HAM, the following Homotopy maps should be constructed as

$$
\begin{aligned}
& H_{I}[\hat{I}(t ; q), \hat{R}(t ; q), \hat{A}(t ; q), \hat{Y}(t ; q), \hat{Z}(t ; q)] \\
& \quad=(1-q) L_{I}\left[\hat{I}(t ; q)-I_{0}(t)\right]-q \hbar H_{I}(t) N_{I}[\hat{I}(t ; q), \hat{R}(t ; q), \hat{A}(t ; q), \hat{Y}(t ; q), \hat{Z}(t ; q)], \\
& H_{R}[\hat{I}(t ; q), \hat{R}(t ; q), \hat{A}(t ; q), \hat{Y}(t ; q), \hat{Z}(t ; q)] \\
& \quad=(1-q) L_{R}\left[\hat{R}(t ; q)-R_{0}(t)\right]-q \hbar H_{R}(t) N_{R}[\hat{I}(t ; q), \hat{R}(t ; q), \hat{A}(t ; q), \hat{Y}(t ; q), \hat{Z}(t ; q)], \\
& H_{A}[\hat{I}(t ; q), \hat{R}(t ; q), \hat{A}(t ; q), \hat{Y}(t ; q), \hat{Z}(t ; q)] \\
& \quad=(1-q) L_{A}\left[\hat{A}(t ; q)-A_{0}(t)\right]-q \hbar H_{A}(t) N_{A}[\hat{I}(t ; q), \hat{R}(t ; q), \hat{A}(t ; q), \hat{Y}(t ; q), \hat{Z}(t ; q)],
\end{aligned}
$$

$$
\begin{aligned}
& H_{Y}[\hat{I}(t ; q), \hat{R}(t ; q), \hat{A}(t ; q), \hat{Y}(t ; q), \hat{Z}(t ; q)] \\
& \quad=(1-q) L_{Y}\left[\hat{Y}(t ; q)-Y_{0}(t)\right]-q \hbar H_{Y}(t) N_{Y}[\hat{I}(t ; q), \hat{R}(t ; q), \hat{A}(t ; q), \hat{Y}(t ; q), \hat{Z}(t ; q)], \\
& H_{Z}[\hat{I}(t ; q), \hat{R}(t ; q), \hat{A}(t ; q), \hat{Y}(t ; q), \hat{Z}(t ; q)] \\
& \quad=(1-q) L_{Z}\left[\hat{Z}(t ; q)-Z_{0}(t)\right]-q \hbar H_{Z}(t) N_{Z}[\hat{I}(t ; q), \hat{R}(t ; q), \hat{A}(t ; q), \hat{Y}(t ; q), \hat{Z}(t ; q)],
\end{aligned}
$$

where $0 \leq q \leq 1$ is an embedding parameter, $\hbar$ is an auxiliary parameter to control the region of convergence, $H_{I}(t), H_{R}(t), H_{A}(t), H_{Y}(t), H_{Z}(t)$ are the auxiliary functions, $L_{I}, L_{R}, L_{A}, L_{Y}, L_{Z}$ are the linear operators and $N_{I}, N_{R}, N_{A}, N_{Y}, N_{Z}$ are the non-linear operators which are defined in the following form 


$$
\begin{aligned}
N_{I}[\hat{I}(t ; q), \hat{R}(t ; q), \hat{A}(t ; q), \hat{Y}(t ; q), \hat{Z}(t ; q)] & =\frac{d \hat{I}(t ; q)}{d t}-m P_{H} \beta_{H} \hat{S}(t ; q) \hat{Z}(t ; q) \\
& +\left(\varepsilon+\mu_{H}\right) \hat{I}(t ; q), \\
N_{R}[\hat{I}(t ; q), \hat{R}(t ; q), \hat{A}(t ; q), \hat{Y}(t ; q), \hat{Z}(t ; q)]= & \frac{d \hat{R}(t ; q)}{d t}-\varepsilon \hat{I}(t ; q)-k \hat{A}(t ; q) \\
& +\left(\theta m P_{H} \beta_{H} \hat{Z}(t ; q)+\tau+\mu_{H}\right) \hat{R}(t ; q), \\
N_{A}[\hat{I}(t ; q), \hat{R}(t ; q), \hat{A}(t ; q), \hat{Y}(t ; q), \hat{Z}(t ; q)]= & \frac{d \hat{A}(t ; q)}{d t}-\theta m P_{H} \beta_{H} \hat{R}(t ; q) \hat{Z}(t ; q) \\
& +\left(k+\mu_{H}\right) \hat{A}(t ; q), \\
N_{Y}[\hat{I}(t ; q), \hat{R}(t ; q), \hat{A}(t ; q), \hat{Y}(t ; q), \hat{Z}(t ; q)] & =\frac{d \hat{Y}(t ; q)}{d t}-P_{H} \beta_{Z}(\hat{I}(t ; q)+\sigma \hat{A}(t ; q)) \hat{X}(t ; q) \\
& +\left(\xi+\mu_{v}\right) \hat{Y}(t ; q), \\
N_{Z}[\hat{I}(t ; q), \hat{R}(t ; q), \hat{A}(t ; q), \hat{Y}(t ; q), \hat{Z}(t ; q)] & =\frac{d \hat{Z}(t ; q)}{d t}-\xi \hat{Y}(t ; q)+\mu_{v} \hat{Z}(t ; q) .
\end{aligned}
$$

Now, we define the zero order deformation equations as follows

$$
\begin{aligned}
& (1-q) L_{I}\left[\hat{I}(t ; q)-I_{0}(t)\right]-q \hbar H_{I}(t) N_{I}[\hat{I}(t ; q), \hat{R}(t ; q), \hat{A}(t ; q), \hat{Y}(t ; q), \hat{Z}(t ; q)]=0 \\
& (1-q) L_{R}\left[\hat{R}(t ; q)-R_{0}(t)\right]-q \hbar H_{R}(t) N_{R}[\hat{I}(t ; q), \hat{R}(t ; q), \hat{A}(t ; q), \hat{Y}(t ; q), \hat{Z}(t ; q)]=0, \\
& (1-q) L_{A}\left[\hat{A}(t ; q)-A_{0}(t)\right]-q \hbar H_{A}(t) N_{A}[\hat{I}(t ; q), \hat{R}(t ; q), \hat{A}(t ; q), \hat{Y}(t ; q), \hat{Z}(t ; q)]=0 \\
& (1-q) L_{Y}\left[\hat{Y}(t ; q)-Y_{0}(t)\right]-q \hbar H_{Y}(t) N_{Y}[\hat{I}(t ; q), \hat{R}(t ; q), \hat{A}(t ; q), \hat{Y}(t ; q), \hat{Z}(t ; q)]=0 \\
& (1-q) L_{Z}\left[\hat{Z}(t ; q)-Z_{0}(t)\right]-q \hbar H_{Z}(t) N_{Z}[\hat{I}(t ; q), \hat{R}(t ; q), \hat{A}(t ; q), \hat{Y}(t ; q), \hat{Z}(t ; q)]=0
\end{aligned}
$$

When parameter $q$ changes from zero to one, the exact solution can be obtained by starting from the initial functions $I_{0}(t), R_{0}(t), A_{0}(t), Y_{0}(t), Z_{0}(t)$. We apply the Taylor polynomials as

$$
\begin{aligned}
& \hat{I}(t ; q)=I_{0}(t)+\sum_{m=1}^{\infty} I_{m}(t) q^{m}, \\
& \hat{R}(t ; q)=R_{0}(t)+\sum_{m=1}^{\infty} R_{m}(t) q^{m}, \\
& \hat{A}(t ; q)=A_{0}(t)+\sum_{m=1}^{\infty} A_{m}(t) q^{m}, \\
& \hat{Y}(t ; q)=Y_{0}(t)+\sum_{m=1}^{\infty} Y_{m}(t) q^{m}, \\
& \hat{Z}(t ; q)=Z_{0}(t)+\sum_{m=1}^{\infty} Z_{m}(t) q^{m},
\end{aligned}
$$


where

$$
\begin{aligned}
& I_{m}=\left.\frac{1}{m !} \frac{\partial^{m} \hat{I}(t ; q)}{\partial q^{m}}\right|_{q=0}, \quad R_{m}=\left.\frac{1}{m !} \frac{\partial^{m} \hat{R}(t ; q)}{\partial q^{m}}\right|_{q=0}, \quad A_{m}=\left.\frac{1}{m !} \frac{\partial^{m} \hat{A}(t ; q)}{\partial q^{m}}\right|_{q=0}, \\
& Y_{m}=\left.\frac{1}{m !} \frac{\partial^{m} \hat{Y}(t ; q)}{\partial q^{m}}\right|_{q=0}, \quad Z_{m}=\left.\frac{1}{m !} \frac{\partial^{m} \hat{Z}(t ; q)}{\partial q^{m}}\right|_{q=0} .
\end{aligned}
$$

Vectors $\hat{I}_{m}(t), \hat{R}_{m}(t), \hat{A}_{m}(t), \hat{Y}_{m}(t)$ and $\hat{Z}_{m}(t)$ can be defined as follows

$$
\begin{aligned}
& \hat{I}_{m}(t)=\left\{I_{0}(t), I_{1}(t), \ldots, I_{m}(t)\right\}, \\
& \hat{R}_{m}(t)=\left\{R_{0}(t), R_{1}(t), \ldots, R_{m}(t)\right\}, \\
& \hat{A}_{m}(t)=\left\{A_{0}(t), A_{1}(t), \ldots, A_{m}(t)\right\}, \\
& \hat{Y}_{m}(t)=\left\{Y_{0}(t), Y_{1}(t), \ldots, Y_{m}(t)\right\}, \\
& \hat{Z}_{m}(t)=\left\{Z_{0}(t), Z_{1}(t), \ldots, Z_{m}(t)\right\} .
\end{aligned}
$$

Now, we can construct the $m$-th order deformation equation by differentiating the zero order deformation Equation (7) with respect to $q$, dividing by $m$ ! and putting $q=0$ as

$$
\begin{aligned}
& L_{I}\left[I_{m}(t)-\chi_{m} I_{m-1}(t)\right]=\hbar H_{I}(t) \Re\left(\vec{I}_{m-1}^{I}, \vec{R}_{m-1}, \vec{A}_{m-1}, \vec{Y}_{m-1}, \vec{Z}_{m-1}\right), \\
& L_{R}\left[R_{m}(t)-\chi_{m} R_{m-1}(t)\right]=\hbar H_{R}(t) \Re\left(\vec{I}_{m-1}^{R}, \vec{R}_{m-1}, \vec{A}_{m-1}, \vec{Y}_{m-1}, \vec{Z}_{m-1}\right), \\
& L_{A}\left[A_{m}(t)-\chi_{m} A_{m-1}(t)\right]=\hbar H_{A}(t) \Re_{m}^{A}\left(\vec{I}_{m-1}, \vec{R}_{m-1}, \vec{A}_{m-1}, \vec{Y}_{m-1}, \vec{Z}_{m-1}\right), \\
& L_{Y}\left[Y_{m}(t)-\chi_{m} Y_{m-1}(t)\right]=\hbar H_{Y}(t) \Re_{m}^{Y}\left(\vec{I}_{m-1}, \vec{R}_{m-1}, \vec{A}_{m-1}, \vec{Y}_{m-1}, \vec{Z}_{m-1}\right), \\
& L_{Z}\left[Z_{m}(t)-\chi_{m} Z_{m-1}(t)\right]=\hbar H_{Z}(t) \Re_{m}^{Z}\left(\vec{I}_{m-1}, \vec{R}_{m-1}, \vec{A}_{m-1}, \vec{Y}_{m-1}, \vec{Z}_{m-1}\right),
\end{aligned}
$$

where $\Re_{m}^{I}, \Re_{m}^{R}, \Re_{m}^{A}, \Re_{m}^{Y}$ and $\Re_{m}^{Z}$ are defined in the following form 


$$
\begin{aligned}
\Re_{m}^{I} & =\mathcal{L}\left[I_{m-1}(t)\right]-\frac{I_{m-1}(0)}{s}+\frac{\left(\varepsilon+\mu_{H}\right)}{s} \mathcal{L}\left[I_{m-1}(t)\right] \\
& -\frac{m P_{H} \beta_{H}}{s} \mathcal{L}\left[Z_{m-1}(t)-\sum_{j=0}^{m-1}\left(I_{j}(t) Z_{m-1-j}(t)+R_{j}(t) Z_{m-1-j}(t)+A_{j}(t) Z_{m-1-j}(t)\right)\right] \\
\Re_{m}^{R} & =\mathcal{L}\left[R_{m-1}(t)\right]-\frac{R_{m-1}(0)}{s}-\frac{\varepsilon}{s} \mathcal{L}\left[I_{m-1}(t)\right]-\frac{k}{s} \mathcal{L}\left[A_{m-1}(t)\right] \\
& +\frac{\theta m P_{H} \beta_{H}}{s} \mathcal{L}\left[\sum_{j=0}^{m-1} Z_{j}(t) R_{m-1-j}(t)\right]+\frac{\left(\tau+\mu_{H}\right)}{s} \mathcal{L}\left[R_{m-1}(t)\right] \\
\Re_{m}^{A} & =\mathcal{L}\left[A_{m-1}(t)\right]-\frac{A_{m-1}(0)}{s}-\frac{\theta m P_{H} \beta_{H}}{s} \mathcal{L}\left[\sum_{j=0}^{m-1} R_{j}(t) Z_{m-1-j}(t)\right]+\frac{\left(k+\mu_{H}\right)}{s} \mathcal{L}\left[A_{m-1}(t)\right], \\
\Re_{m}^{Y} & =\mathcal{L}\left[Y_{m-1}(t)\right]-\frac{Y_{m-1}(0)}{s}-\frac{P_{H} \beta_{Z}}{s} \mathcal{L}\left[\sum_{j=0}^{m-1} I_{j}\left(1-Y_{m-1-j}(t)-Z_{m-1-j}(t)\right)\right] \\
& -\frac{P_{H} \beta_{Z} \sigma}{s} \mathcal{L}\left[\sum_{j=0}^{m-1} A_{j}(t)\left(1-Y_{m-1-j}(t)-Z_{m-1-j}(t)\right)\right]+\frac{\left(\xi+\mu_{v}\right)}{s} \mathcal{L}\left[Y_{m-1}(t)\right], \\
\Re_{m}^{Z}= & \mathcal{L}\left[Z_{m-1}(t)\right]-\frac{Z_{m-1}(0)}{s}-\frac{\xi}{s} \mathcal{L}\left[Y_{m-1}(t)\right]+\frac{\mu_{v}}{s} \mathcal{L}\left[Z_{m-1}(t)\right]
\end{aligned}
$$

and

$$
\chi_{m}= \begin{cases}0, & m \leq 1 \\ 1, & m>1 .\end{cases}
$$

By using the inverse Laplace transformation $\mathcal{L}^{-1}$ for Equation (9), the iterative formulas

$$
\begin{aligned}
& I_{m}=\chi_{m} I_{m-1}+\hbar \mathcal{L}^{-1}\left[\Re_{m}^{I}\right], \\
& R_{m}=\chi_{m} R_{m-1}+\hbar \mathcal{L}^{-1}\left[\Re_{m}^{R}\right], \\
& A_{m}=\chi_{m} A_{m-1}+\hbar \mathcal{L}^{-1}\left[\Re_{m}^{A}\right], \\
& Y_{m}=\chi_{m} Y_{m-1}+\hbar \mathcal{L}^{-1}\left[\Re_{m}^{Y}\right], \\
& Z_{m}=\chi_{m} Z_{m-1}+\hbar \mathcal{L}^{-1}\left[\Re_{m}^{Z}\right],
\end{aligned}
$$

and finally the $m$-th order approximate solutions can be estimated by

$$
\begin{array}{ll}
I_{m}(t)=\sum_{j=0}^{m} I_{j}(t), & R_{m}(t)=\sum_{j=0}^{m} R_{j}(t), \quad A_{m}(t)=\sum_{j=0}^{m} A_{j}(t), \\
Y_{m}(t)=\sum_{j=0}^{m} Y_{j}(t), & Z_{m}(t)=\sum_{j=0}^{m} Z_{j}(t) .
\end{array}
$$


The convergence theorem of the HATM for solving the mentioned model is presented in the following form:

Theorem 1. If series solutions (13) obtained from Equation (9) are convergent, then they must be the exact solution of system (3).

Proof. Let the series solutions (13) be convergent [12-14]. If

$$
\begin{aligned}
& P_{1}(t)=\sum_{m=0}^{\infty} I_{m}(t), \quad P_{2}(t)=\sum_{m=0}^{\infty} R_{m}(t), \quad P_{3}(t)=\sum_{m=0}^{\infty} A_{m}(t), \\
& P_{4}(t)=\sum_{m=0}^{\infty} Y_{m}(t), \quad P_{5}(t)=\sum_{m=0}^{\infty} Z_{m}(t),
\end{aligned}
$$

then

$$
\begin{aligned}
& \lim _{m \rightarrow \infty} I_{m}(t)=0, \quad \lim _{m \rightarrow \infty} R_{m}(t)=0, \quad \lim _{m \rightarrow \infty} A_{m}(t)=0, \\
& \lim _{m \rightarrow \infty} Y_{m}(t)=0, \quad \lim _{m \rightarrow \infty} Z_{m}(t)=0 .
\end{aligned}
$$

For left hand side of $m$-th order deformation Equation (9) we get

$$
\begin{aligned}
& \sum_{m=1}^{N}\left[I_{m}(t)-\chi_{m} I_{m-1}(t)\right]=I_{N}(t) \\
& \sum_{m=1}^{N}\left[R_{m}(t)-\chi_{m} R_{m-1}(t)\right]=R_{N}(t), \\
& \sum_{m=1}^{N}\left[A_{m}(t)-\chi_{m} A_{m-1}(t)\right]=A_{N}(t), \\
& \sum_{m=1}^{N}\left[Y_{m}(t)-\chi_{m} Y_{m-1}(t)\right]=Y_{N}(t), \\
& \sum_{m=1}^{N}\left[Z_{m}(t)-\chi_{m} Z_{m-1}(t)\right]=Z_{N}(t) .
\end{aligned}
$$

Now, Equations (15) and (16) are applied as

$$
\begin{aligned}
& \sum_{m=1}^{N}\left[I_{m}(t)-\chi_{m} I_{m-1}(t)\right]=\lim _{N \rightarrow \infty} I_{N}(t)=0 \\
& \sum_{m=1}^{N}\left[R_{m}(t)-\chi_{m} R_{m-1}(t)\right]=\lim _{N \rightarrow \infty} R_{N}(t)=0 \\
& \sum_{m=1}^{N}\left[A_{m}(t)-\chi_{m} A_{m-1}(t)\right]=\lim _{N \rightarrow \infty} A_{N}(t)=0, \\
& \sum_{m=1}^{N}\left[Y_{m}(t)-\chi_{m} Y_{m-1}(t)\right]=\lim _{N \rightarrow \infty} Y_{N}(t)=0 \\
& \sum_{m=1}^{N}\left[Z_{m}(t)-\chi_{m} Z_{m-1}(t)\right]=\lim _{N \rightarrow \infty} Z_{N}(t)=0 .
\end{aligned}
$$


By using the linear operators $L_{I}, L_{R}, L_{A}, L_{Y}$, and $L_{Z}$ we can write

$$
\begin{aligned}
& \sum_{m=1}^{\infty} L_{I}\left[I_{m}(t)-\chi_{m} I_{m-1}(t)\right]=L_{I}\left[\sum_{m=1}^{\infty} I_{m}(t)-\chi_{m} I_{m-1}(t)\right]=0 \\
& \sum_{m=1}^{\infty} L_{R}\left[R_{m}(t)-\chi_{m} R_{m-1}(t)\right]=L_{R}\left[\sum_{m=1}^{\infty} R_{m}(t)-\chi_{m} R_{m-1}(t)\right]=0 \\
& \sum_{m=1}^{\infty} L_{A}\left[A_{m}(t)-\chi_{m} A_{m-1}(t)\right]=L_{A}\left[\sum_{m=1}^{\infty} A_{m}(t)-\chi_{m} A_{m-1}(t)\right]=0 \\
& \sum_{m=1}^{\infty} L_{Y}\left[Y_{m}(t)-\chi_{m} Y_{m-1}(t)\right]=L_{Y}\left[\sum_{m=1}^{\infty} Y_{m}(t)-\chi_{m} Y_{m-1}(t)\right]=0 \\
& \sum_{m=1}^{\infty} L_{Z}\left[Z_{m}(t)-\chi_{m} Z_{m-1}(t)\right]=L_{Z}\left[\sum_{m=1}^{\infty} Z_{m}(t)-\chi_{m} Z_{m-1}(t)\right]=0
\end{aligned}
$$

so the following formulas can be obtained

$$
\begin{aligned}
& \hbar H_{I}(t) \sum_{m=1}^{\infty} \Re_{m}^{I}\left(\vec{I}_{m-1}, \vec{R}_{m-1}, \vec{A}_{m-1}, \vec{Y}_{m-1}, \vec{Z}_{m-1}\right)=0, \\
& \hbar H_{R}(t) \sum_{m=1}^{\infty} \Re_{m}^{R}\left(\vec{I}_{m-1}, \vec{R}_{m-1}, \vec{A}_{m-1}, \vec{Y}_{m-1}, \vec{Z}_{m-1}\right)=0, \\
& \hbar H_{A}(t) \sum_{m=1}^{\infty} \Re_{m}^{A}\left(\vec{I}_{m-1}, \vec{R}_{m-1}, \vec{A}_{m-1}, \vec{Y}_{m-1}, \vec{Z}_{m-1}\right)=0, \\
& \hbar H_{Y}(t) \sum_{m=1}^{\infty} \Re_{m}^{Y}\left(\vec{I}_{m-1}, \vec{R}_{m-1}, \vec{A}_{m-1}, \vec{Y}_{m-1}, \vec{Z}_{m-1}\right)=0, \\
& \hbar H_{Z}(t) \sum_{m=1}^{\infty} \Re_{m}^{Z}\left(\vec{I}_{m-1}, \vec{R}_{m-1}, \vec{A}_{m-1}, \vec{Y}_{m-1}, \vec{Z}_{m-1}\right)=0 .
\end{aligned}
$$

Since in Equations (19), $\hbar, H_{I}(t), H_{R}(t), H_{A}(t), H_{Y}(t), H_{Z}(t) \neq 0$, we have

$$
\begin{aligned}
& \sum_{m=1}^{\infty} \Re_{m}^{I}\left(\vec{I}_{m-1}, \vec{R}_{m-1}, \vec{A}_{m-1}, \vec{Y}_{m-1}, \vec{Z}_{m-1}\right)=0, \\
& \sum_{m=1}^{\infty} \Re_{m}^{R}\left(\vec{I}_{m-1}, \vec{R}_{m-1}, \vec{A}_{m-1}, \vec{Y}_{m-1}, \vec{Z}_{m-1}\right)=0, \\
& \sum_{m=1}^{\infty} \Re_{m}^{A}\left(\vec{I}_{m-1}, \vec{R}_{m-1}, \vec{A}_{m-1}, \vec{Y}_{m-1}, \vec{Z}_{m-1}\right)=0, \\
& \sum_{m=1}^{\infty} \Re_{m}^{Y}\left(\vec{I}_{m-1}, \vec{R}_{m-1}, \vec{A}_{m-1}, \vec{Y}_{m-1}, \vec{Z}_{m-1}\right)=0, \\
& \sum_{m=1}^{\infty} \Re_{m}^{Z}\left(\vec{I}_{m-1}, \vec{R}_{m-1}, \vec{A}_{m-1}, \vec{Y}_{m-1}, \vec{Z}_{m-1}\right)=0 .
\end{aligned}
$$

By putting $\Re_{m}^{I}, \Re_{m}^{R}, \Re_{m}^{A}, \Re_{m}^{Y}$ and $\Re_{m}^{Z}$ into Equations (17) and denoting $(.)^{\prime}=\frac{d}{d t}$ we get 


$$
\begin{aligned}
\sum_{m=1}^{\infty} \Re_{m}^{I}=\sum_{m=1}^{\infty}\left[I_{m-1}^{\prime}(t)+\left(\varepsilon+\mu_{H}\right) I_{m-1}(t)-m P_{H} \beta_{H}\left[Z_{m-1}(t)\right.\right. & \\
& \left.\left.-\sum_{j=0}^{m-1}\left(I_{j}(t) Z_{m-1-j}(t)+R_{j}(t) Z_{m-1-j}(t)+A_{j}(t) Z_{m-1-j}(t)\right)\right]\right] \\
= & \sum_{m=0}^{\infty} I_{m}^{\prime}(t)+\left(\varepsilon+\mu_{H}\right) \sum_{m=0}^{\infty} I_{m}(t)-m P_{H} \beta_{H}\left[\sum_{m=0}^{\infty} Z_{m}(t)\right. \\
& \left.-\sum_{m=1}^{\infty} \sum_{j=0}^{m-1}\left(I_{j}(t) Z_{m-1-j}(t)+R_{j}(t) Z_{m-1-j}(t)+A_{j}(t) Z_{m-1-j}(t)\right)\right] \\
= & \sum_{m=0}^{\infty} I_{m}^{\prime}(t)+\left(\varepsilon+\mu_{H}\right) \sum_{m=0}^{\infty} I_{m}(t)-m P_{H} \beta_{H}\left[\sum_{m=0}^{\infty} Z_{m}(t)\right. \\
& \left.-\sum_{j=0}^{\infty} \sum_{m=j+1}^{\infty}\left(I_{j}(t) Z_{m-1-j}(t)+R_{j}(t) Z_{m-1-j}(t)+A_{j}(t) Z_{m-1-j}(t)\right)\right] \\
= & \sum_{m=0}^{\infty} I_{m}^{\prime}(t)+\left(\varepsilon+\mu_{H}\right) \sum_{m=0}^{\infty} I_{m}(t)-m P_{H} \beta_{H}\left[\sum_{m=0}^{\infty} Z_{m}(t)\right. \\
& \left.-\left(\sum_{j=0}^{\infty} I_{j}(t) \sum_{m=0}^{\infty} Z_{m}(t)+\sum_{j=0}^{\infty} R_{j}(t) \sum_{m=0}^{\infty} Z_{m}(t)+\sum_{j=0}^{\infty} A_{j}(t) \sum_{m=0}^{\infty} Z_{m}(t)\right)\right] \\
= & P_{1}^{\prime}(t)+\left(\varepsilon+\mu_{H}\right) P_{1}(t)-m P_{H} \beta_{H}\left[P_{5}(t)-\left(P_{1}(t) P_{5}(t)+P_{2}(t) P_{5}(t)+P_{3}(t) P_{5}(t)\right)\right],
\end{aligned}
$$


and

$$
\begin{aligned}
& \sum_{m=1}^{\infty} \Re_{m}^{R}=\sum_{m=1}^{\infty}\left[R_{m-1}^{\prime}(t)-\varepsilon I_{m-1}(t)-k A_{m-1}(t)\right. \\
& \left.\quad+\theta m P_{H} \beta_{H} \sum_{j=0}^{m-1} Z_{j}(t) R_{m-1-j}(t)+\left(\tau+\mu_{H}\right) R_{m-1}(t)\right] \\
& =\sum_{m=0}^{\infty} R_{m}^{\prime}(t)-\varepsilon \sum_{m=0}^{\infty} I_{m}(t)-k \sum_{m=0}^{\infty} A_{m}(t) \\
& \quad+\theta m P_{H} \beta_{H} \sum_{m=1}^{\infty} \sum_{j=0}^{m-1} Z_{j}(t) R_{m-1-j}(t)+\left(\tau+\mu_{H}\right) \sum_{m=0}^{\infty} R_{m}(t) \\
& =\sum_{m=0}^{\infty} R_{m}^{\prime}(t)-\varepsilon \sum_{m=0}^{\infty} I_{m}(t)-k \sum_{m=0}^{\infty} A_{m}(t) \\
& \quad+\theta m P_{H} \beta_{H} \sum_{j=0}^{\infty} \sum_{m=j+1}^{\infty} Z_{j}(t) R_{m-1-j}(t)+\left(\tau+\mu_{H}\right) \sum_{m=0}^{\infty} R_{m}(t) \\
& =\sum_{m=0}^{\infty} R_{m}^{\prime}(t)-\varepsilon \sum_{m=0}^{\infty} I_{m}(t)-k \sum_{m=0}^{\infty} A_{m}(t) \\
& \quad+\theta m P_{H} \beta_{H} \sum_{j=0}^{\infty} Z_{j}(t) \sum_{m=0}^{\infty} R_{m}(t)+\left(\tau+\mu_{H}\right) \sum_{m=0}^{\infty} R_{m}(t) \\
& =P_{2}^{\prime}(t)-\varepsilon P_{1}(t)-k P_{3}(t)+\theta m P_{H} \beta_{H} P_{5}(t) P_{2}(t)+\left(\tau+\mu_{H}\right) P_{2}(t),
\end{aligned}
$$

and

$$
\begin{aligned}
& \sum_{m=1}^{\infty} \Re_{m}^{A}=\sum_{m=1}^{\infty}\left[A_{m-1}^{\prime}(t)-\theta m P_{H} \beta_{H} \sum_{j=0}^{m-1} R_{j}(t) Z_{m-1-j}(t)+\left(k+\mu_{H}\right) A_{m-1}(t)\right] \\
& =\sum_{m=0}^{\infty} A_{m}^{\prime}(t)-\theta m P_{H} \beta_{H} \sum_{m=1}^{\infty} \sum_{j=0}^{m-1} R_{j}(t) Z_{m-1-j}(t)+\left(k+\mu_{H}\right) \sum_{m=0}^{\infty} A_{m}(t) \\
& =\sum_{m=0}^{\infty} A_{m}^{\prime}(t)-\theta m P_{H} \beta_{H} \sum_{j=0}^{\infty} \sum_{m=j+1}^{\infty} R_{j}(t) Z_{m-1-j}(t)+\left(k+\mu_{H}\right) \sum_{m=0}^{\infty} A_{m}(t) \\
& =\sum_{m=0}^{\infty} A_{m}^{\prime}(t)-\theta m P_{H} \beta_{H} \sum_{j=0}^{\infty} R_{j}(t) \sum_{m=0}^{\infty} Z_{m}(t)+\left(k+\mu_{H}\right) \sum_{m=0}^{\infty} A_{m}(t) \\
& =P_{3}^{\prime}(t)-\theta m P_{H} \beta_{H} P_{2}(t) P_{5}(t)+\left(k+\mu_{H}\right) P_{3}(t),
\end{aligned}
$$

and 


$$
\begin{aligned}
& \sum_{m=1}^{\infty} \Re_{m}^{Y}=\sum_{m=1}^{\infty}\left[Y_{m-1}^{\prime}(t)-P_{H} \beta_{Z}\left[\sum_{j=0}^{m-1} I_{j}\left(1-Y_{m-1-j}(t)-Z_{m-1-j}(t)\right)\right]\right. \\
& \left.-P_{H} \beta_{Z} \sigma\left(\sum_{j=0}^{m-1} A_{j}(t)\left(1-Y_{m-1-j}(t)-Z_{m-1-j}(t)\right)\right)+\left(\xi+\mu_{v}\right) Y_{m-1}(t)\right] \\
& =\sum_{m=0}^{\infty} Y_{m}^{\prime}(t)-P_{H} \beta_{Z}\left[\sum_{m=1}^{\infty} \sum_{j=0}^{m-1} I_{j}\left(1-Y_{m-1-j}(t)-Z_{m-1-j}(t)\right)\right] \\
& -P_{H} \beta_{Z} \sigma\left(\sum_{m=1}^{\infty} \sum_{j=0}^{m-1} A_{j}(t)\left(1-Y_{m-1-j}(t)-Z_{m-1-j}(t)\right)\right)+\left(\xi+\mu_{v}\right) \sum_{m=0}^{\infty} Y_{m}(t) \\
& =\sum_{m=0}^{\infty} Y_{m}^{\prime}(t)-P_{H} \beta_{Z}\left[\sum_{j=0}^{\infty} \sum_{m=j+1}^{\infty} I_{j}\left(1-Y_{m-1-j}(t)-Z_{m-1-j}(t)\right)\right] \\
& -P_{H} \beta_{Z} \sigma\left(\sum_{j=0}^{\infty} \sum_{m=j+1}^{\infty} A_{j}(t)\left(1-Y_{m-1-j}(t)-Z_{m-1-j}(t)\right)\right)+\left(\xi+\mu_{v}\right) \sum_{m=0}^{\infty} Y_{m}(t) \\
& =\sum_{m=0}^{\infty} Y_{m}^{\prime}(t)-P_{H} \beta_{Z}\left[\sum_{j=0}^{\infty} I_{j} \sum_{m=0}^{\infty}\left(1-Y_{m}(t)-Z_{m}(t)\right)\right] \\
& -P_{H} \beta_{Z} \sigma\left(\sum_{j=0}^{\infty} A_{j}(t) \sum_{m=0}^{\infty}\left(1-Y_{m}(t)-Z_{m}(t)\right)\right)+\left(\xi+\mu_{v}\right) \sum_{m=0}^{\infty} Y_{m}(t) \\
& =P_{4}^{\prime}(t)-P_{H} \beta_{Z} P_{1}(t)\left(1-P_{4}(t)-P_{5}(t)\right)-P_{H} \beta_{Z} \sigma P_{3}(t)\left(1-P_{4}(t)-P_{5}(t)\right)+\left(\xi+\mu_{v}\right) P_{4}(t), \\
& \text { and finally } \\
& \sum_{m=1}^{\infty} \Re_{m}^{Z_{a}}=\sum_{m=1}^{\infty}\left[Z_{m-1}^{\prime}(t)-\xi Y_{m-1}(t)+\mu_{v} Z_{m-1}(t)\right]=\sum_{m=0}^{\infty} Z_{m}^{\prime}(t)-\xi \sum_{m=0}^{\infty} Y_{m}(t)+\mu_{v} \sum_{m=0}^{\infty} Z_{m}(t) \\
& =P_{5}^{\prime}(t)-\xi P_{4}(t)+\mu_{v} P_{5}(t) .
\end{aligned}
$$

Equations (21)-(25) show that the series solutions $P_{1}(t), P_{2}(t), P_{3}(t), P_{4}(t)$ and $P_{5}(t)$ must be the exact solutions of the system of Equations (3).

\section{CESTAC Method-CADNA Library}

As we know, usual numerical and semi analytical methods for solving mathematical and engineering problems are based on the FPA. However, because of the mentioned problems, it will be better if we replace the FPA by a random arithmetic. For this aim, we apply the DSA which has many applications and more advantages than other arithmetics. Thus, we apply the CESTAC method and the CADNA library to validate the numerical results. For more information about this method please see [21,23].

By collecting all representable values which are produced by computer in set $B$, we can write $S^{*} \in B$ for $s^{*} \in \mathbb{R}$ with $\alpha$ mantissa bits of the binary FPA as

$$
S^{*}=s^{*}-\rho 2^{E-\alpha} \phi \text {, }
$$

where sign, missing segment of the mantissa and the binary exponent of the result are denoted by $\rho, 2^{-\alpha} \phi$ and $E$ respectively. In order to find the results with single and double precisions, the value $\alpha$ can be changed to 24 and 53. Let $\phi$ be a casual variable that is 
uniformly distributed on $[-1,1]$. If we make perturbation on $s^{*}$, then $(\mu)$ and $(\sigma)$ can be provided as mean and standard deviation of the results. Iterating this procedure $k$ times, we can obtain $\mu \simeq s^{*}$. Algorithm 1 shows the mentioned process, where $\tau_{\delta}$ is the value of the $T$ distribution as the confidence interval is $1-\delta$, with $k-1$ degrees of freedom $[22,24,25]$.

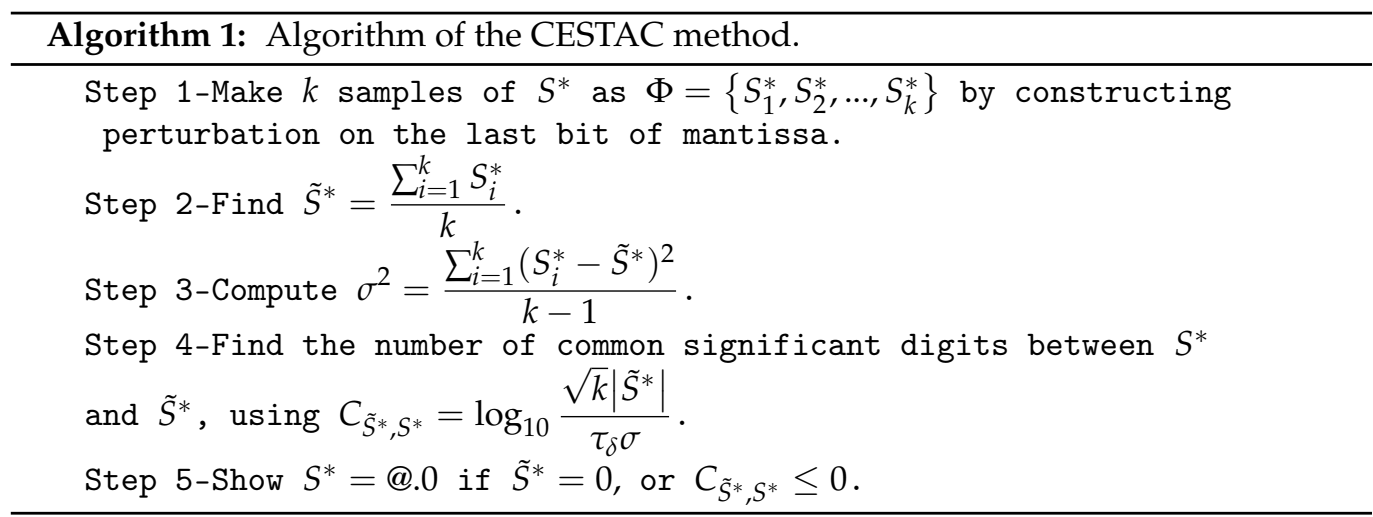

When we want to apply the CESTAC method, instead of using the algorithm directly, we should apply the CADNA library. The library can implement the algorithm automatically. The CADNA library should be done on Linux operating system and all codes should be improved by $\mathrm{C}, \mathrm{C}++$, FORTRAN or ADA codes. So in this method we do not need to apply the usual mathematical softwares such as Mathematica, Maple and MATLAB. Applying the CESTAC method and DSA we have some advantages in comparison with the methods based on the FPA. In order to apply the termination criterion (1) which is based on the FPA we need to have the exact solution but in the DSA we do not need the exact solution and the stopping condition (2) is based on two successive approximations [21]. In the FPA, we do not know the optimal $\varepsilon$ and in the DSA we do not have value $\varepsilon$. In the FPA, the extra iterations can be produced without improving the accuracy, but in the DSA we can find the optimal number of iterations. In the FPA, the algorithm can be stopped in the first step without producing the accurate results but in the DSA, the optimal approximation can be identified. In the CESTAC method, we can produce @.0 which shows the number of common significant digits between two successive approximations are zero but in the FPA we can not produce this sign [27-29]. Next, we have the sample code of the CADNA library:

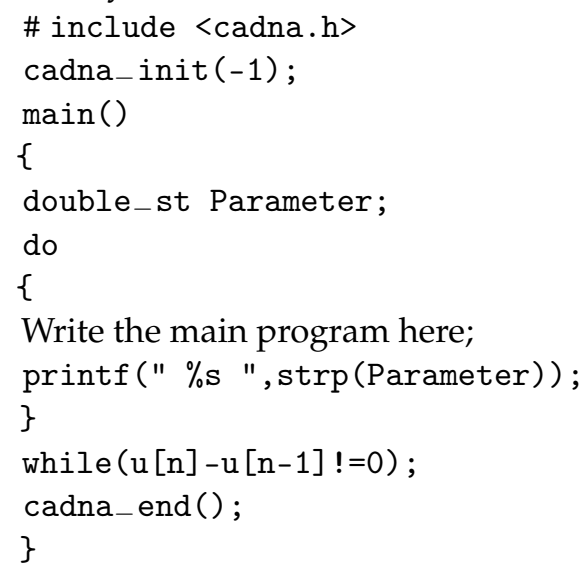


Definition 1. Ref. [21] For two real numbers $\Psi_{1}$ and $\Psi_{2}$, the number of common significant digits can be defined as

$$
\left\{\begin{array}{l}
C_{\Psi_{1}, \Psi_{2}}=\log _{10}\left|\frac{\Psi_{1}+\Psi_{2}}{2\left(\Psi_{1}-\Psi_{2}\right)}\right|=\log _{10}\left|\frac{\Psi_{1}}{\Psi_{1}-\Psi_{2}}-\frac{1}{2}\right|, \quad \Psi_{1} \neq \Psi_{2}, \\
C_{\Psi_{1}, \Psi_{1}}=+\infty
\end{array}\right.
$$

Theorem 2. Let the approximate solutions of the mathematical model of Malaria infection (3) be obtained using series (13) which are produced by the HATM. Then

$$
\begin{aligned}
& C_{I_{m}, I_{m+1}}=C_{I_{m}, I}+O\left(\frac{1}{m}\right), \\
& C_{R_{m}, R_{m+1}}=C_{R_{m}, R}+O\left(\frac{1}{m}\right), \\
& C_{A_{m}, A_{m+1}}=C_{A_{m}, A}+O\left(\frac{1}{m}\right), \\
& C_{Y_{m}, Y_{m+1}}=C_{Y_{m}, Y}+O\left(\frac{1}{m}\right), \\
& C_{Z_{m}, Z_{m+1}}=C_{Z_{m}, Z}+O\left(\frac{1}{m}\right),
\end{aligned}
$$

Proof. Using Definition 1 we have

$$
\begin{aligned}
C_{I_{m}, I_{m+1}}-C_{I_{m}, I} & =\log _{10}\left|\frac{I_{m}+I_{m+1}}{2\left(I_{m}-I_{m+1}\right)}\right|-\log _{10}\left|\frac{I_{m}+I}{2\left(I_{m}-I\right)}\right| \\
& =\log _{10}\left|\frac{I_{m}+I_{m+1}}{2\left(I_{m+1}^{\hbar}\right)}\right|-\log _{10}\left|\frac{I_{m}+I}{2\left(I_{m}-I\right)}\right| \\
& =\log _{10}\left|\frac{I_{m}+I_{m+1}}{I_{m}+I}\right|+\log _{10}\left|\frac{I_{m}-I}{I_{m+1}^{\hbar}}\right| .
\end{aligned}
$$

By increasing the iterations number $m$, we can see in the first term of Equation (29), the approximate and exact solutions $I_{m}, I$ are close together and we can neglect that. For the second term we have

$$
\begin{aligned}
\log _{10}\left|\frac{I_{m}-I}{I_{m+1}}\right| & =\log _{10}\left|\frac{\sum_{i=0}^{m} I_{i}-\sum_{i=0}^{\infty} I_{i}}{I_{m+1}}\right| \\
& =\log _{10}\left|\frac{\sum_{i=m+1}^{\infty} I_{i}}{I_{m+1}}\right| \\
& =\log _{10}\left|1+\frac{\sum_{i=m+2}^{\infty} \hbar L^{-1}\left[H(r) \Re_{i}\left(u_{i-1}\right)\right]}{\hbar L^{-1}\left[H(r) \Re_{m+1}\left(u_{m}\right)\right]}\right| .
\end{aligned}
$$


Now, we can write

$$
\begin{aligned}
& \frac{\sum_{i=m+2}^{\infty} \hbar L^{-1}\left[H(r) \Re\left(u_{i-1}\right)\right]}{\hbar L^{-1}\left[H(r) \Re_{m+1}\left(u_{m}\right)\right]}=\frac{\sum_{i=m+2}^{\infty} \hbar L^{-1}\left[\left.H(r) \frac{1}{(i-1) !} \frac{\partial^{i-1} N[U(r, v)]}{\partial v^{i-1}}\right|_{v=0}\right]}{\hbar L^{-1}\left[\left.H(r) \frac{1}{m !} \frac{\partial^{m} N[U(r, v)]}{\partial v^{m}}\right|_{v=0}\right]} \\
& =\frac{\hbar L^{-1}\left[\left.H(r) \frac{1}{(m+1) !} \frac{\partial^{m+1} N[U(r, v)]}{\partial v^{m+1}}\right|_{v=0}\right]}{\hbar L^{-1}\left[\left.H(r) \frac{1}{m !} \frac{\partial^{m} N[U(r, v)]}{\partial v^{m}}\right|_{v=0}\right]}+\frac{\hbar L^{-1}\left[\left.H(r) \frac{1}{(m+2) !} \frac{\partial^{m+2} N[U(r, v)]}{\partial v^{m+2}}\right|_{v=0}\right]}{\hbar L^{-1}\left[\left.H(r) \frac{1}{m !} \frac{\partial^{m} N[U(r, v)]}{\partial v^{m}}\right|_{v=0}\right]}+\cdots \\
& =O\left(\frac{1}{m}\right) .
\end{aligned}
$$

Therefore, we get

$$
\log _{10}\left|\frac{I_{m}-I}{I_{m+1}}\right|=\log _{10}\left|1+O\left(\frac{1}{m}\right)\right| .
$$

By repeating the process for other terms we have

$$
\begin{aligned}
& \log _{10}\left|\frac{R_{m}-R}{R_{m+1}}\right|=\log _{10}\left|1+O\left(\frac{1}{m}\right)\right|, \\
& \log _{10}\left|\frac{A_{m}-A}{A_{m+1}}\right|=\log _{10}\left|1+O\left(\frac{1}{m}\right)\right|, \\
& \log _{10}\left|\frac{Y_{m}-Y}{Y_{m+1}}\right|=\log _{10}\left|1+O\left(\frac{1}{m}\right)\right|, \\
& \log _{10}\left|\frac{Z_{m}-Z}{Z_{m+1}}\right|=\log _{10}\left|1+O\left(\frac{1}{m}\right)\right| .
\end{aligned}
$$

Since $O\left(\frac{1}{m}\right)<<1$, then the right hand side of the above relation decreases as $m$ increases. Thus, the proof is complete.

\section{Numerical Illustration}

In this section, the numerical results of the nonlinear model of malaria infection (3) are presented using the HATM. The numerical results for $m=5$ are obtained as follows 


$$
\begin{aligned}
& I_{5}(t)=5+1.80089 \hbar t+3.60178 \hbar^{2} t+3.60178 \hbar^{3} t+1.80089 \hbar^{4} t+0.360178 \hbar^{5} t \\
& -0.10765 \hbar^{2} t^{2}-0.215299 \hbar^{3} t^{2}-0.161474 \hbar^{4} t^{2}-0.0430598 \hbar^{5} t^{2}-0.0242739 \hbar^{3} t^{3} \\
& -0.0364109 \hbar^{4} t^{3}-0.0145644 \hbar^{5} t^{3}-0.000774695 \hbar^{4} t^{4}-0.000619756 \hbar^{5} t^{4} \\
& -5.05807 \times 10^{-6} \hbar^{5} t^{5} ; \\
& R_{5}(t)=15+0.13655 \hbar t+0.273099 \hbar^{2} t+0.273099 \hbar^{3} t+0.13655 \hbar^{4} t+0.0273099 \hbar^{5} t \\
& -0.0426064 \hbar^{2} t^{2}-0.0852128 \hbar^{3} t^{2}-0.0639096 \hbar^{4} t^{2}-0.0170426 \hbar^{5} t^{2}-0.00584539 \hbar^{3} t^{3} \\
& -0.00876809 \hbar^{4} t^{3}-0.00350723 \hbar^{5} t^{3}-0.000159503 \hbar^{4} t^{4}-0.000127602 \hbar^{5} t^{4} \\
& -9.33113 \times 10^{-7} \hbar^{5} t^{5} ;
\end{aligned}
$$$$
\begin{aligned}
& A_{5}(t)=10-0.180842 \hbar t-0.361684 \hbar^{2} t-0.361684 \hbar^{3} t-0.180842 \hbar^{4} t-0.0361684 \hbar^{5} t \\
& +0.0337365 \hbar^{2} t^{2}+0.0674729 \hbar^{3} t^{2}+0.0506047 \hbar^{4} t^{2}+0.0134946 \hbar^{5} t^{2}+0.00601047 \hbar^{3} t^{3} \\
& +0.0090157 \hbar^{4} t^{3}+0.00360628 \hbar^{5} t^{3}+0.000173945 \hbar^{4} t^{4}+0.000139156 \hbar^{5} t^{4} \\
& +1.0817 \times 10^{-6} \hbar^{5} t^{5} ;
\end{aligned}
$$$$
Y_{5}(t)=100+88.5228 \hbar t+177.046 \hbar^{2} t+177.046 \hbar^{3} t+88.5228 \hbar^{4} t+17.7046 \hbar^{5} t
$$$$
+13.96 \hbar^{2} t^{2}+27.9199 \hbar^{3} t^{2}+20.9399 \hbar^{4} t^{2}+5.58399 \hbar^{5} t^{2}+0.582255 \hbar^{3} t^{3}
$$$$
+0.873382 \hbar^{4} t^{3}+0.349353 \hbar^{5} t^{3}+0.00252142 \hbar^{4} t^{4}+0.00201714 \hbar^{5} t^{4}-0.000106061 \hbar^{5} t^{5} \text {; }
$$$$
\begin{aligned}
& Z_{5}(t)=55-22.5 \hbar t-45 . \hbar^{2} t-45 . \hbar^{3} t-22.5 \hbar^{4} t-4.5 \hbar^{5} t-11.1023 \hbar^{2} t^{2}-22.2046 \hbar^{3} t^{2} \\
& -16.6534 \hbar^{4} t^{2}-4.44091 \hbar^{5} t^{2}-0.835408 \hbar^{3} t^{3}-1.25311 \hbar^{4} t^{3}-0.501245 \hbar^{5} t^{3} \\
& -0.0177208 \hbar^{4} t^{4}-0.0141766 \hbar^{5} t^{4}-0.0000809688 \hbar^{5} t^{5}
\end{aligned}
$$

It is obvious that the obtained solution of the HATM is based on $t$ and $\hbar$. The auxiliary parameter $\hbar$ is an independent variable and we can plot the graph versus $\hbar$. Assume that

$$
\begin{aligned}
& \Gamma_{I}=\left.I(t ; q)\right|_{q=0, t=1}, \quad \text { or } \quad \Gamma_{I}^{\prime}=\left.I^{\prime}(t ; q)\right|_{q=0, t=1}, \\
& \Gamma_{R}=\left.R(t ; q)\right|_{q=0, t=1}, \quad \text { or } \Gamma_{R}^{\prime}=\left.R^{\prime}(t ; q)\right|_{q=0, t=1}, \\
& \Gamma_{A}=\left.A(t ; q)\right|_{q=0, t=1}, \quad \text { or } \quad \Gamma_{A}^{\prime}=\left.A^{\prime}(t ; q)\right|_{q=0, t=1}, \\
& \Gamma_{Y}=\left.Y(t ; q)\right|_{q=0, t=1}, \quad \text { or } \quad \Gamma_{Y}^{\prime}=\left.Y^{\prime}(t ; q)\right|_{q=0, t=1}, \\
& \Gamma_{Z}=\left.Z(t ; q)\right|_{q=0, t=1}, \quad \text { or } \quad \Gamma_{Z}^{\prime}=\left.Z^{\prime}(t ; q)\right|_{q=0, t=1},
\end{aligned}
$$

thus the curves $\Gamma$ are functions of $\hbar$ and the graph of $\Gamma \approx \hbar$ can be plotted where these regions are called the valid convergence regions. According to $[12,13]$ there exists a horizontal line segment (flat portion of the $\hbar$-curve) in the figures $\Gamma \approx \hbar$ to find the convergence regions. Therefore, by choosing any values of $\hbar$ in the valid regions, we can be sure that the corresponding solutions are convergent. For given initial parameters and functions the valid regions of $\hbar$ can be obtained often nearly the same for a given problem. Thus, plotting the $\hbar$-curves will be a suitable way to show the convergence regions of the problem. In Figures 1 and 2, the $\hbar$-curves for $t=1$ and $m=5,10$ are plotted. According to these graphs, the convergence regions for $m=5$ are

$$
\begin{aligned}
& -1.4 \leq \hbar_{I, 5} \leq-0.8 \\
& -1.2 \leq \hbar_{R, 5} \leq-0.8 \\
& -1.2 \leq \hbar_{A, 5} \leq-0.6 \\
& -1.2 \leq \hbar_{Y, 5} \leq-0.6, \\
& -1.2 \leq \hbar_{Z, 5} \leq-0.7,
\end{aligned}
$$


and for $m=10$ are

$$
\begin{aligned}
& -1.3 \leq \hbar_{I, 10} \leq-0.6, \\
& -1.3 \leq \hbar_{R, 10} \leq-0.6, \\
& -1.3 \leq \hbar_{A, 10} \leq-0.6, \\
& -1.3 \leq \hbar_{Y, 10} \leq-0.6, \\
& -1.2 \leq \hbar_{Z, 10} \leq-0.6 .
\end{aligned}
$$

Figure 3 shows the residual error functions based on $\hbar$ for $t=1$ and $m=5,10$. Based on this graph, we can find the region $\hbar$ for minimizing the error function. In Figure 4 , the residual error functions based on $t$ for $\hbar=-1$ and $m=5,10$ are depicted which shows the accuracy of the method. The main aim of this study is to compare the numerical results based on the FPA and the SA. Table 2 shows the residual errors based on the FPA for $\hbar=-1$ and $m=5,10$. In Table 3, the numerical results are based on the FPA and the termination criterion (1). Thus, we need to have the value $\varepsilon$. It is clear that for $\varepsilon=10^{-4}$ the HATM is stopped at $m=4$ without providing accurate results. In Table 4 , the number of iterations of the method for different values of $\varepsilon$ are presented. It is obvious that for large values $\varepsilon$ we cannot provide the accurate results and the method is stopped very soon. For small values of $\varepsilon$, we also have large number of iterations. Because of these disadvantages, we apply the DSA, the CESTAC method and the CADNA library to validate the results. In Table 5 the numerical results are obtained based on the CESTAC method and applying the stopping condition (2) as follows

$$
\begin{aligned}
& I_{\text {opt }}=4.63133024427036, \\
& R_{\text {opt }}=14.9689830310043, \\
& A_{\text {opt }}=10.0389756223080, \\
& Y_{\text {opt }}=83.6337838483667, \\
& Z_{\text {opt }}=58.4698479078703,
\end{aligned}
$$

where the optimal iteration of the HATM for solving model (3) is $m_{\text {opt }}=11$. According to the CADNA report, for solving this method using the HATM we have 84 numerical instabilities including 2 unstable intrinsic functions and 82 loss of accuracy to cancellation.

Table 2. The residual error based on the FPA for $\hbar=-1$ and $m=5,10$.

\begin{tabular}{ccccccc}
\hline $\boldsymbol{m}$ & $\boldsymbol{t}$ & $\boldsymbol{I}(\boldsymbol{t})$ & $\boldsymbol{R}(\boldsymbol{t})$ & $\boldsymbol{A}(\boldsymbol{t})$ & $\boldsymbol{Y}(\boldsymbol{t})$ & $\boldsymbol{Z}(\boldsymbol{t})$ \\
\hline \multirow{4}{*}{5} & 0.0 & $4.44089 \times 10^{-16}$ & $3.46945 \times 10^{-17}$ & $2.08167 \times 10^{-17}$ & $7.10543 \times 10^{-15}$ & 0 \\
& 0.2 & $1.81148 \times 10^{-11}$ & $1.81942 \times 10^{-12}$ & $9.61586 \times 10^{-12}$ & $1.51737 \times 10^{-8}$ & $8.0294 \times 10^{-10}$ \\
& 0.4 & $4.12406 \times 10^{-10}$ & $7.92373 \times 10^{-11}$ & $3.28725 \times 10^{-10}$ & $4.8434 \times 10^{-7}$ & $2.5694 \times 10^{-8}$ \\
& 0.6 & $1.89596 \times 10^{-9}$ & $7.5749 \times 10^{-10}$ & $2.65204 \times 10^{-9}$ & $3.66829 \times 10^{-6}$ & $1.95114 \times 10^{-7}$ \\
& 0.8 & $2.92335 \times 10^{-9}$ & $3.83283 \times 10^{-9}$ & $1.18164 \times 10^{-8}$ & 0.0000154157 & $8.22209 \times 10^{-7}$ \\
& 1.0 & $6.1194 \times 10^{-9}$ & $1.36056 \times 10^{-8}$ & $3.79696 \times 10^{-8}$ & 0.000046911 & $2.50918 \times 10^{-6}$ \\
\hline \multirow{4}{*}{10} & 0.0 & $1.26565 \times 10^{-14}$ & $3.67761 \times 10^{-16}$ & $1.04777 \times 10^{-15}$ & $6.32383 \times 10^{-13}$ & 0 \\
& 0.2 & $1.21569 \times 10^{-14}$ & $8.04912 \times 10^{-16}$ & $7.56339 \times 10^{-16}$ & $4.90274 \times 10^{-13}$ & $2.66454 \times 10^{-14}$ \\
& 0.4 & $1.17684 \times 10^{-14}$ & $1.25594 \times 10^{-15}$ & $5.55112 \times 10^{-16}$ & $3.33955 \times 10^{-13}$ & $9.76996 \times 10^{-14}$ \\
& 0.6 & $8.21565 \times 10^{-15}$ & $2.42861 \times 10^{-15}$ & $9.47159 \times 10^{-16}$ & $2.27374 \times 10^{-13}$ & $1.45661 \times 10^{-13}$ \\
& 0.8 & $3.94129 \times 10^{-14}$ & $6.88338 \times 10^{-15}$ & $4.19109 \times 10^{-15}$ & $2.84217 \times 10^{-12}$ & $6.85674 \times 10^{-13}$ \\
& 1.0 & $4.71623 \times 10^{-13}$ & $4.91551 \times 10^{-14}$ & $4.41314 \times 10^{-14}$ & $2.88338 \times 10^{-11}$ & $7.90834 \times 10^{-12}$ \\
\hline
\end{tabular}



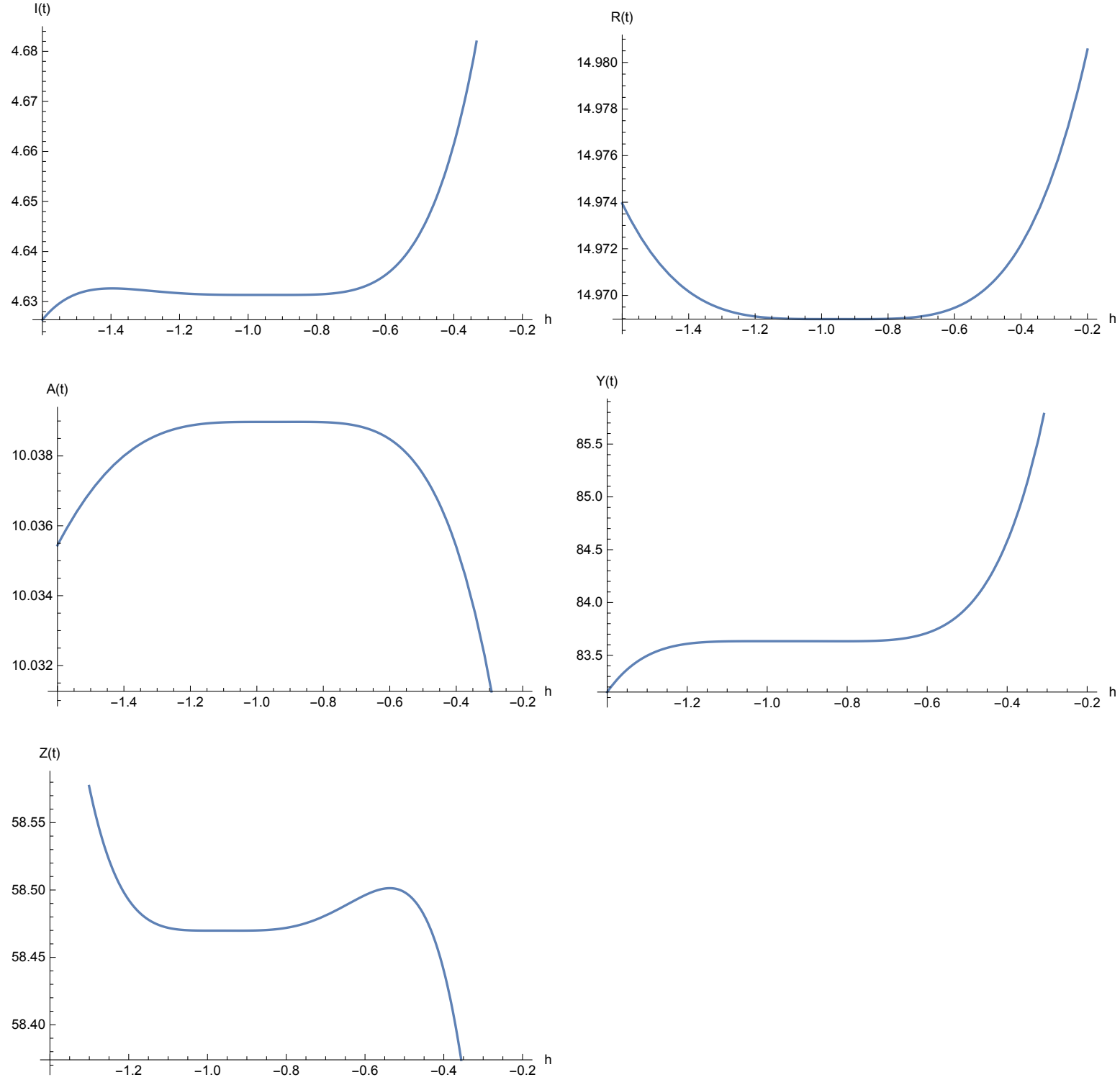

Figure 1. The $\hbar$-curves for $t=1$ and $m=5$. 

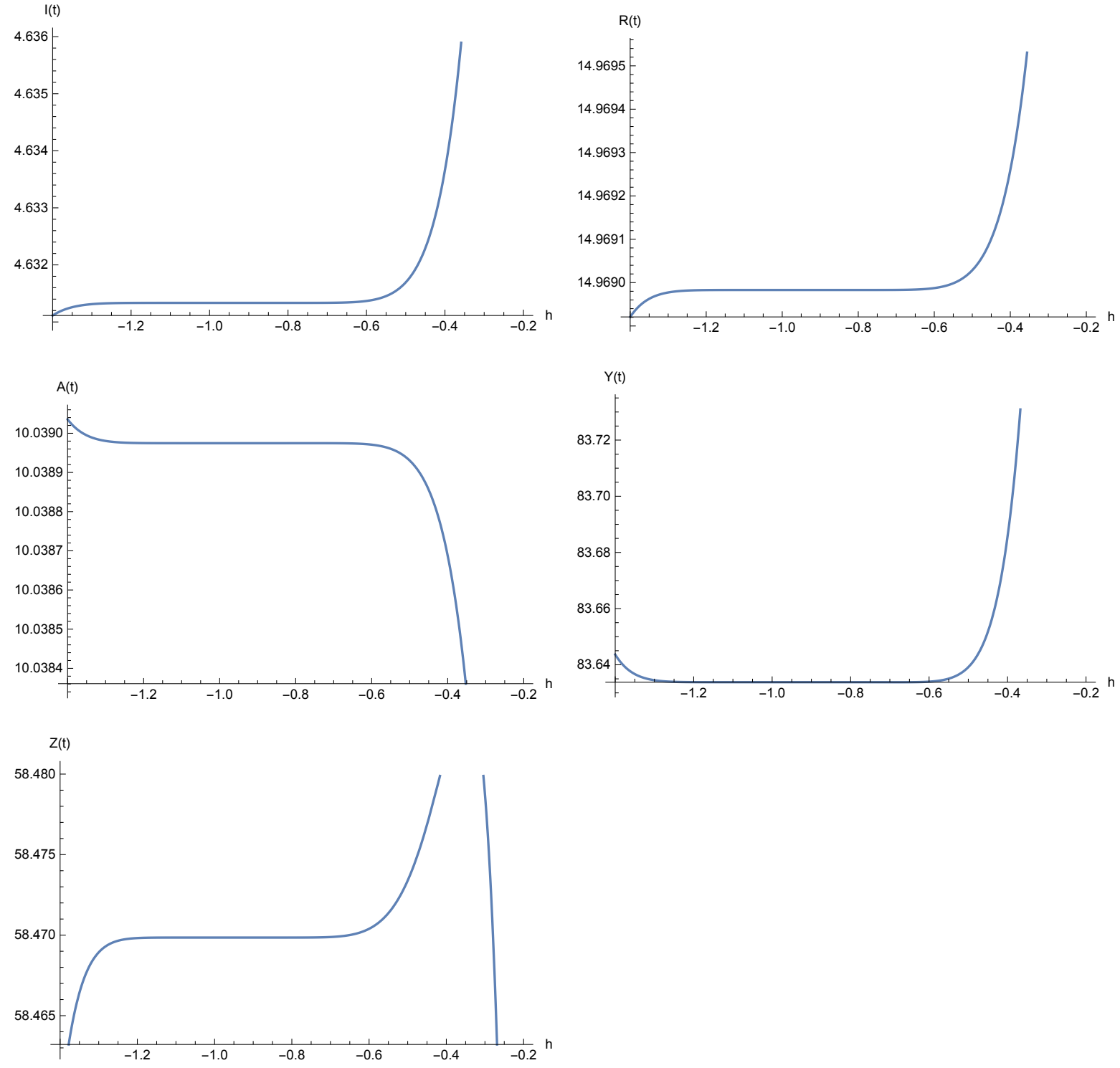

Figure 2. The $\hbar$-curves for $t=1$ and $m=10$.
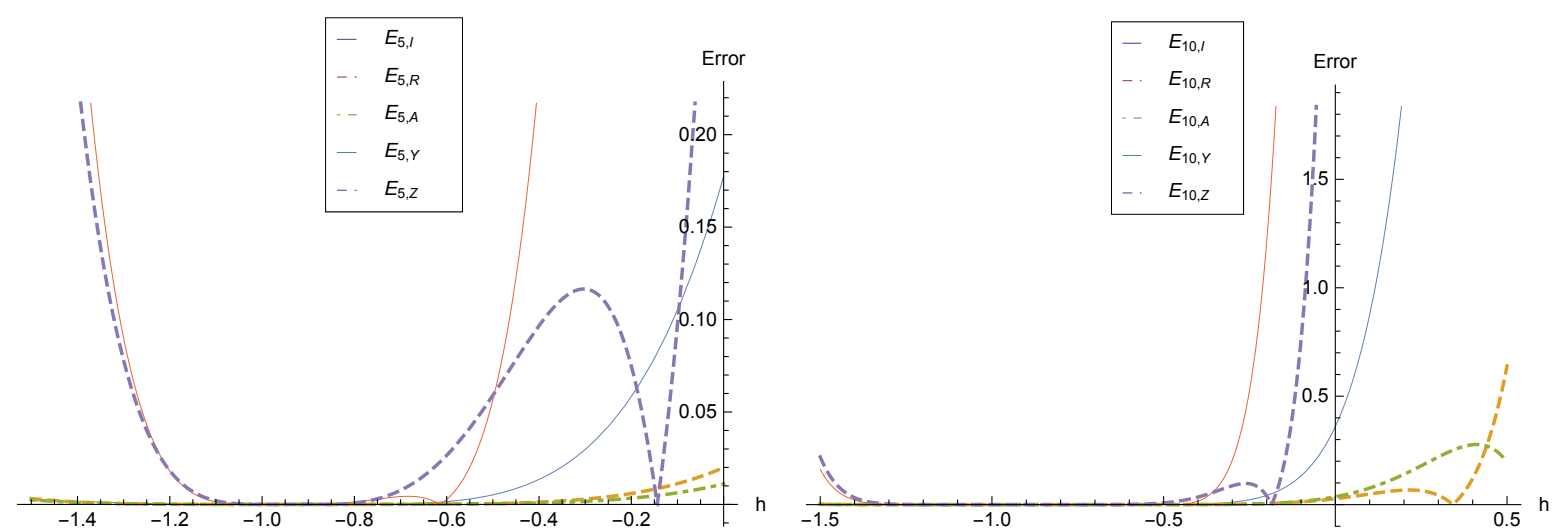

Figure 3. Graph of residual error functions based on $\hbar$ for $t=1$ and $m=5,10$. 

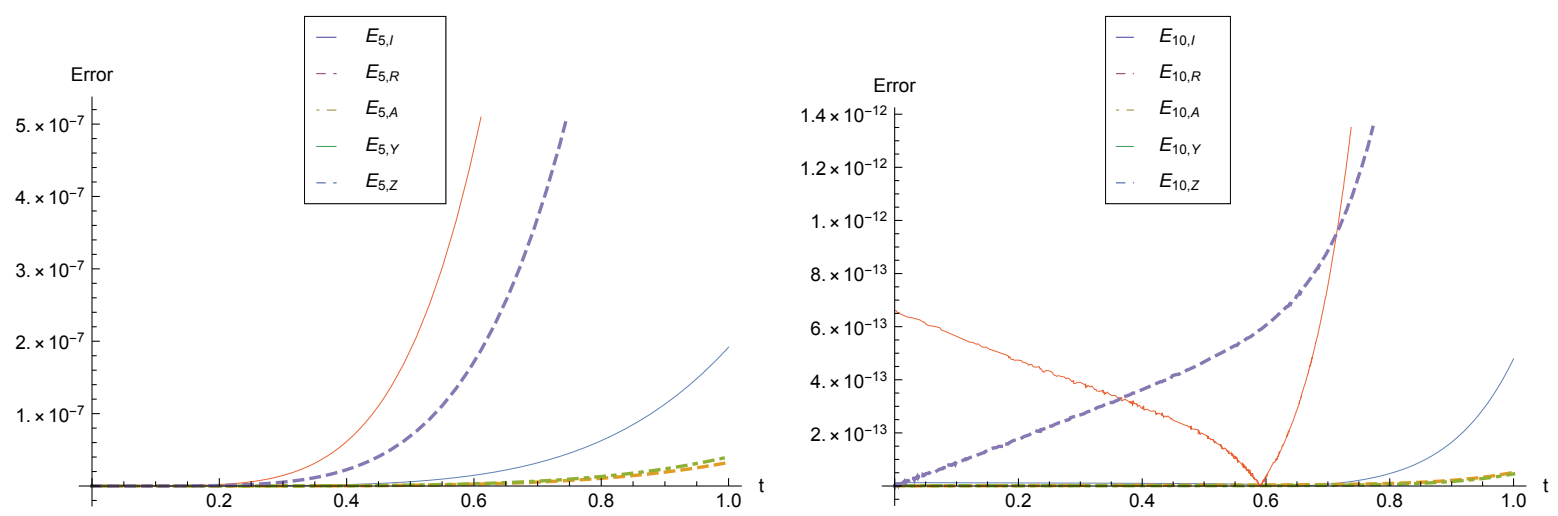

Figure 4. Graph of residual error functions based on $t$ for $\hbar=-1$ and $m=5,10$.

Table 3. The numerical results based on the FPA and condition (1) for $t=1, \hbar=-1$ and $\varepsilon=10^{-4}$.

\begin{tabular}{lll}
\hline$m$ & Approximate Solutions & Difference between Two Iterations \\
\hline 1 & 4.639822 & 4.639822 \\
1 & 14.9726900999999 & 14.9726900999999 \\
1 & 10.0361684000000 & 10.0361684 \\
1 & 82.2954000000000 & 82.2954 \\
1 & 59.4999999999999 & 59.4999999999999 \\
\hline 2 & 4.629057 & $0.1076499999999 \times 10^{-1}$ \\
2 & 14.96842946 & $0.426063999999 \times 10^{-2}$ \\
2 & 10.0395420499999 & $0.337364999999 \times 10^{-2}$ \\
2 & 83.6913999999999 & 13.959999999999 \\
2 & 58.3897699999999 & 11.102299999999 \\
\hline 3 & 4.63148439 & $0.242738999999 \times 10^{-2}$ \\
3 & 14.969013999 & $0.58453899999 \times 10^{-3}$ \\
3 & 10.0389410029999 & $0.60104699999 \times 10^{-3}$ \\
3 & 83.6331745 & $0.582255000000 \times 10^{-1}$ \\
3 & 58.4733108 & $0.8354079999999 \times 10^{-1}$ \\
\hline 4 & 4.631329451 & $0.15493900000 \times 10^{-3}$ \\
4 & 14.9689820983999 & $0.3190060000 \times 10^{-4}$ \\
4 & 10.0389757918999 & $0.3478889999 \times 10^{-4}$ \\
4 & 83.6336787849999 & $0.5042850000 \times 10^{-3}$ \\
4 & 58.46976664 & $0.354415999999 \times 10^{-2}$ \\
\hline
\end{tabular}

Table 4. The number of iterations for different values of $\varepsilon$ based on the FPA.

\begin{tabular}{ccccccc}
\hline$\varepsilon$ & Small Values & $\varepsilon=10^{-13}$ & $\varepsilon=10^{-6}$ & $\varepsilon=10^{-1}$ & $\varepsilon=0.5$ & Large Values \\
\hline$m$ & $>>10$ & 10 & 5 & 2 & 1 & 1 \\
\hline
\end{tabular}


Table 5. The numerical results using the CESTAC method for $t=1$ and $\hbar=-1$.

\begin{tabular}{|c|c|c|}
\hline$m$ & Approximate Solutions & Difference between Two Iterations \\
\hline 1 & 4.63982200000000 & 0.4 .63982200000000 \\
\hline 1 & 14.9726900999999 & 0.14 .9726900999999 \\
\hline 1 & 10.0361684000000 & 0.10 .0361684000000 \\
\hline 1 & 82.2954000000000 & 0.82 .2954000000000 \\
\hline 1 & 59.4999999999999 & 0.59 .4999999999999 \\
\hline 2 & 4.62905700000000 & $0.1076499999999 \times 10^{-1}$ \\
\hline 2 & 14.9684294600000 & $0.426063999999 \times 10^{-2}$ \\
\hline 2 & 10.0395420499999 & $0.337364999999 \times 10^{-2}$ \\
\hline 2 & 83.6913999999999 & 1.3959999999999 \\
\hline 2 & 58.3897699999999 & 1.1102299999999 \\
\hline 3 & 4.63148439000000 & $0.242738999999 \times 10^{-2}$ \\
\hline 3 & 14.9690139990000 & $0.58453899999 \times 10^{-3}$ \\
\hline 3 & 10.0389410029999 & $0.60104699999 \times 10^{-3}$ \\
\hline 3 & 83.6331745000000 & $0.582255000000 \times 10^{-1}$ \\
\hline 3 & 58.4733108000000 & $0.8354079999999 \times 10^{-1}$ \\
\hline 4 & 4.63132945100000 & $0.15493900000 \times 10^{-3}$ \\
\hline 4 & 14.9689820983999 & $0.3190060000 \times 10^{-4}$ \\
\hline 4 & 10.0389757918999 & $0.3478889999 \times 10^{-4}$ \\
\hline 4 & 83.6336787849999 & $0.5042850000 \times 10^{-3}$ \\
\hline 4 & 58.4697666400000 & $0.354415999999 \times 10^{-2}$ \\
\hline 5 & 4.63133024369753 & $0.79269753 \times 10^{-6}$ \\
\hline 5 & 14.9689830315129 & $0.93311300 \times 10^{-6}$ \\
\hline 5 & 10.0389756223766 & $0.1695233 \times 10^{-6}$ \\
\hline 5 & 83.6337848460000 & $0.106061000 \times 10^{-3}$ \\
\hline 5 & 58.4698476088000 & $0.809688000 \times 10^{-4}$ \\
\hline 6 & 4.63133025286105 & $0.916351 \times 10^{-8}$ \\
\hline 6 & 14.9689830309158 & $0.59716 \times 10^{-9}$ \\
\hline 6 & 10.0389756224041 & $0.2748 \times 10^{-10}$ \\
\hline 6 & 83.6337836044895 & $0.1241510 \times 10^{-5}$ \\
\hline 6 & 58.4698480269969 & $0.4181970 \times 10^{-6}$ \\
\hline 7 & 4.63133024345624 & $0.940480 \times 10^{-8}$ \\
\hline 7 & 14.9689830309063 & $0.944 \times 10^{-11}$ \\
\hline 7 & 10.0389756224136 & $0.950 \times 10^{-11}$ \\
\hline 7 & 83.6337838482735 & $0.2437839 \times 10^{-6}$ \\
\hline 7 & 58.4698479078530 & $0.1191439 \times 10^{-6}$ \\
\hline 8 & 4.63133024430048 & $0.84423 \times 10^{-9}$ \\
\hline 8 & 14.9689830310048 & $0.98461 \times 10^{-10}$ \\
\hline 8 & 10.0389756223075 & $0.1061 \times 10^{-9}$ \\
\hline 8 & 83.6337838483026 & $0.291 \times 10^{-10}$ \\
\hline 8 & 58.4698479078797 & $0.267 \times 10^{-10}$ \\
\hline 9 & 4.63133024427004 & $0.3043 \times 10^{-10}$ \\
\hline 9 & 14.9689830310043 & $0.47 \times 10^{-12}$ \\
\hline 9 & 10.0389756223080 & $0.5 \times 10^{-12}$ \\
\hline 9 & 83.6337838482963 & $0.63 \times 10^{-11}$ \\
\hline 9 & 58.4698479078804 & $0.68 \times 10^{-12}$ \\
\hline
\end{tabular}


Table 5. Cont.

\begin{tabular}{lll}
\hline $\boldsymbol{m}$ & Approximate Solutions & Difference between Two Iterations \\
\hline 10 & $4.63133024427030 \mathrm{E}+001$ & $0.25 \times 10^{-12}$ \\
10 & 14.9689830310043 & $0.2 \times 10^{-13}$ \\
10 & 10.0389756223080 & $0.4 \times 10^{-13}$ \\
10 & 83.6337838483671 & $0.708 \times 10^{-10}$ \\
10 & 58.4698479078696 & $0.10 \times 10^{-10}$ \\
\hline 11 & 4.63133024427036 & $0.6 \times 10^{-13}$ \\
11 & 14.9689830310043 & $@ .0$ \\
11 & 10.0389756223080 & $@ .0$ \\
11 & 83.6337838483667 & $0.4 \times 10^{-12}$ \\
11 & 58.4698479078703 & $0.74 \times 10^{-12}$ \\
\hline
\end{tabular}

\title{
6. Conclusions
}

In this paper, we focused on solving the nonlinear bio-mathematical model of malaria infection. Because of reflexibility of the HATM, we applied this method to find the approximate solutions of the problem. We have some disadvantages when we want to apply the mathematical methods based on the FPA. Thus we prefer to use the DSA, which has many advantages. For this aim, the CESTAC method and the CADNA library were proposed. Using this method we can find the optimal approximations, optimal iteration and the numerical instabilities of the method. In the CESTAC method, we also apply a new termination criterion (2) instead of the traditional conditions (1). In the new technique we do not need to have the exact solution and also the positive value $\varepsilon$. The convergence theorem of the HATM to solve the model was proved. Moreover we focused on proving the main theorem of the CESTAC method. Thus, we can apply the condition (2) instead of the previous one. Plotting several $\hbar$-curves we found the valid convergence region of the solution. We also presented the numerical results based on both DSA and FPA to show the abilities of the DSA. The FPA results depend on the value $\varepsilon$ such that for large values of $\varepsilon$ we do not have the accurate results and for small values we have extra iterations without improving the accuracy. The DSA results are based on two successive iterations and we do not need to have value $\varepsilon$. As our future studies, we will focus on the fractional model of Malaria infection applying the DSA.

Author Contributions: Conceptualization, S.N. and S.M.; Data curation, S.N.; Formal analysis, S.N. and S.M.; Funding acquisition, S.N. and S.M.; Investigation, S.N.; Methodology, S.N. and S.M.; Project administration, S.N.; Resources, S.N. and S.M.; Software, S.N.; Supervision, S.N.; Validation, S.N.; Visualization, S.N.; Writing—original draft, S.N. and S.M.; Writing—review \& editing, S.N. and S.M. All authors have read and agreed to the published version of the manuscript.

Funding: This research received no external funding.

Institutional Review Board Statement: Not applicable.

Informed Consent Statement: Not applicable.

Data Availability Statement: Not applicable.

Conflicts of Interest: The authors declare no conflict of interest.

\author{
Abbreviations \\ The following abbreviations are used in this manuscript: \\ HATM Homotopy Analysis Transform Method \\ FPA Floating Point Arithmetic \\ DSA Discrete Stochastic Arithmetic \\ CESTAC Controle et Estimation Stochastique des Arrondis de Calculs \\ CADNA Control of Accuracy and Debugging for Numerical Applications
}




\section{References}

1. Noeiaghdam, S.; Sidorov, D. Caputo-Fabrizio Fractional Derivative to Solve the Fractional Model of Energy Supply-Demand System. Math. Model. Eng. Probl. 2020, 7, 359-367. [CrossRef]

2. Naik, P.A.; Zu, J.; Ghoreishi, M. Stability analysis and approximate solution of SIR epidemic model with Crowley-Martin type functional response and holling type-II treatment rate by using homotopy analysis method. J. Appl. Anal. Comput. 2020, 10, 1482-1515.

3. Noeiaghdam, S.; Khoshrouye Ghiasi, E. An efficient method to solve the mathematical model of HIV infection for CD8+T cells. Int. J. Math. Model. Comput. 2019, 36, 267-281.

4. Naik, P.A.; Owolabi, K.M.; Yavuz, M.; Zu, J. Chaotic dynamics of a fractional order HIV-1 model involving AIDS-related cancer cells. Chaos Solitons Fractals 2020, 140, 110272.

5. Naik, P.A.; Zu, J.; Ghoreishi, M. Estimating the approximate analytical solution of HIV viral dynamic model by using homotopy analysis method. Chaos Solitons Fractals 2020, 131, 109500. [CrossRef]

6. Noeiaghdam, S.; Kamal Ali, K. Semi-analytical Method to Solve the Non-linear System of Equations to Model of Evolution for Smoking Habit in Spain. Int. J. Ind. Math. 2020, 12, 1377.

7. Ahmad, A.; Farman, M.; Naik, P.A.; Akgul, A.; Zafar, N.; Saleem, M.U. Modeling and numerical investigation of fractional-order bovine babesiosis disease. Numer. Methods Partial. Differ. Equ. 2021, 37, 1946-1964. [CrossRef]

8. Noeiaghdam, S. Numerical Approximation of Modified Non-linear SIR Model of Computer Viruses. Contemp. Math. 2019, 1, 10804. [CrossRef]

9. Noeiaghdam, S. A novel technique to solve the modified epidemiological model of computer viruses. SeMA J. 2019, 76, 97-108.

10. Noeiaghdam, S.; Suleman, M.; Budak, H. Solving a modified non-linear epidemiological model of computer viruses by homotopy analysis method. Math. Sci. 2018, 12, 211-222. [CrossRef]

11. Overview. Available online: https://www.who.int/health-topics/malaria\#tab=tab_1 (accessed on 15 February 2021).

12. Liao, S.J. Beyond Perturbation: Introduction to Homotopy Analysis Method; Chapman \& Hall/CRC Press: Boca Raton, FL, USA, 2003.

13. Liao, S.J. Homotopy Analysis Method in Nonlinear Differential Equations; Higher Education Press: Beijing, China; Springer: Berlin/Heidelberg, Germany, 2012.

14. Liao, S.J. The Proposed Homotopy Analysis Techniques for the Solution of Nonlinear Problems. Ph.D. Thesis, Shanghai Jiao Tong University, Shanghai, China, 1992.

15. Fariborzi Araghi, M.A.; Noeiaghdam, S. Homotopy analysis transform method for solving generalized Abel's fuzzy integral equations of the first kind. Iran. Jt. Congr. Fuzzy Intell. Syst. CFIS 2015, 2016, 7391645. [CrossRef]

16. Noeiaghdam, S.; Fariborzi Araghi, M.A. Homotopy regularization method to solve the singular Volterra integral equations of the first kind. Jordan J. Math. Stat. 2018, 11, 1-12.

17. Fariborzi Araghi, M.A.; Noeiaghdam, S. A novel technique based on the homotopy analysis method to solve the first kind Cauchy integral equations arising in the theory of airfoils. J. Interpolat. Approx. Sci. Comput. 2016, 2016, 1-13. [CrossRef]

18. Noeiaghdam, S.; Zarei, E.; Barzegar Kelishami, H. Homotopy analysis transform method for solving Abel's integral equations of the first kind. Ain Shams Eng. J. 2016, 7, 483-495. [CrossRef]

19. Khoshrouye Ghiasi, E.; Noeiaghdam, S. Truncating the series expansion for unsteady velocity-dependent Eyring-Powell fluid. Eng. Appl. Sci. Lett. 2020, 3, 28-34. [CrossRef]

20. Nave, O.; Lehavi, Y.; Gol'dshtein, V.; Ajadi, S. Application of the Homotopy Perturbation Method (HPM) and the Homotopy Analysis Method (HAM) to the Problem of the Thermal Explosion in a Radiation Gas with Polydisperse Fuel Spray. J. Appl. Comput. Math. 2012, 1. [CrossRef]

21. Fariborzi Araghi, M.A.; Noeiaghdam, S. Validation of Numerical Algorithms: Stochastic Arithmetic; Entekhab Bartar Publisher: Tehran, Iran, 2021; ISBN 978-622-6498-09-8.

22. Chesneaux, J.M. CADNA, an ADA tool for round-off error analysis and for numerical debugging. In Proceedings of the Congress on ADA, Aerospace, Barcelona, 20-25 May 1990.

23. Laboratoire d'Informatique de Paris 6. Available online: https://www-pequan.lip6.fr/ (accessed on 10 December 2020).

24. Chesneaux, J.M.; Jézéquel, F. Dynamical control of computations using the Trapezoidal and Simpson's rules. J. Univers. Comput. Sci. 1998, 4, 2-10.

25. Graillat, S.; Jézéquel, F.; Wang, S.; Zhu, Y. Stochastic arithmetic in multi precision. Math. Comput. Sci. 2011, 5, 359-375. [CrossRef]

26. Graillat, S.; Jézéquel, F.; Picot, R. Numerical Validation of Compensated Summation Algorithms with Stochastic Arithmetic. Electron. Notes Theor. Comput. Sci. 2015, 317, 55-69.

27. Jézéquel, F.; Mecanique, C.R. A dynamical strategy for approximation methods. Comptes Rendus Mec. 2006, 334, 362-367. [CrossRef]

28. Vignes, J. Discrete Stochastic Arithmetic for Validating Results of Numerical Software. Spec. Issue Numer. Algorithms 2004, 7 , 377-390. [CrossRef]

29. Vignes, J. A stochastic arithmetic for reliable scientific computation. Math. Comput. Simul. 1993, 35, 233-261. [CrossRef]

30. Noeiaghdam, S.; Fariborzi Araghi, M.A. A novel algorithm to evaluate definite integrals by the Gauss-Legendre integration rule based on the stochastic arithmetic: Application in the model of osmosis system. Math. Model. Eng. Probl. 2020, 7, 577-586. [CrossRef] 
31. Fariborzi Araghi, M.A.; Noeiaghdam, S. Dynamical control of computations using the Gauss-Laguerre integration rule by applying the CADNA library. Adv. Appl. Math. Sci. 2016, 16, 1-18.

32. Noeiaghdam, S.; Fariborzi Araghi, M.A.; Abbasbandy, S. Finding optimal convergence control parameter in the homotopy analysis method to solve integral equations based on the stochastic arithmetic. Numer. Algorithms 2019, 81, 237-267. [CrossRef]

33. Noeiaghdam, S.; Fariborzi Araghi, M.A. Application of the CESTAC Method to Find the Optimal Iteration of the Homotopy Analysis Method for Solving Fuzzy Integral Equations. Adv. Intell. Syst. Comput. 2021, 1301.

34. Noeiaghdam, L.; Noeiaghdam, S.; Sidorov, D. Dynamical Control on the Homotopy Analysis Method for Solving Nonlinear Shallow Water Wave Equation. J. Phys. Conf. Ser. 1847, 012010. [CrossRef]

35. Noeiaghdam, S.; Dreglea, A.; He, J.H.; Avazzadeh, Z.; Suleman, M.; Fariborzi Araghi, M.A.; Sidorov, D.; Sidorov, N. Error estimation of the homotopy perturbation method to solve second kind Volterra integral equations with piecewise smooth kernels: Application of the CADNA library. Symmetry 2020, 12, 1730. [CrossRef]

36. Noeiaghdam, S.; Sidorov, D.; Wazwaz, A.M.; Sidorov, N.; Sizikov, V. The numerical validation of the Adomian decomposition method for solving Volterra integral equation with discontinuous kernel using the CESTAC method. Mathematics 2021, 9, 260. [CrossRef]

37. Noeiaghdam, S.; Fariborzi Araghi, M.A.; Abbasbandy, S. Valid implementation of Sinc-collocation method to solve the fuzzy Fredholm integral equation. J. Comput. Appl. Math. 2020, 370, 112632. [CrossRef]

38. Noeiaghdam, S.; Sidorov, D.; Sizikov, V.; Sidorov, N. Control of accuracy on Taylor-collocation method to solve the weakly regular Volterra integral equations of the first kind by using the CESTAC method. Appl. Comput. Math. Int. J. 2020, 19, 81-105.

39. Noeiaghdam, S.; Fariborzi Araghi, M.A. Valid implementation of the Sinc-collocation method to solve the linear integral equations by CADNA library. J. Math. Model. 2019, 7, 63-84. [CrossRef]

40. Fariborzi Araghi, M.A.; Noeiaghdam, S. Finding the optimal step of fuzzy Newton-Cotes integration rules by using CESTAC method. J. Fuzzy Set Valued Anal. 2017, 2, 62-85.

41. Fariborzi Araghi, M.A.; Noeiaghdam, S. A valid scheme to evaluate fuzzy definite integrals by applying the CADNA library. Int. J. Fuzzy Syst. Appl. 2017, 6, 1-20.

42. Noeiaghdam, S.; Sidorov, D.; Muftahov, I.; Zhukov, A.V. Control of Accuracy on Taylor-Collocation Method for Load Leveling Problem. Bull. Irkutsk State Univ. Ser. Math. 2019, 30, 59-72. [CrossRef]

43. Noeiaghdam, S.; Sidorov, D.; Zamyshlyaeva, A.; Tynda, A.; Dreglea, A. A valid dynamical control on the reverse osmosis system using the CESTAC method. Mathematics 2021, 9, 48. [CrossRef]

44. Noeiaghdam, S.; Fariborzi Araghi, M.A. A novel approach to find optimal parameter in the homotopy-regularization method for solving integral equations. Appl. Math. Inf. Sci. 2020, 14, 105-113. [CrossRef]

45. Yakob, L. Endectocide-treated cattle for malaria control: A coupled entomological-epidemiological model. Parasite Epidemiol. Control 2016, 1, 2-9. [CrossRef] 